Fig. 1 Top-the number of uses of 291 ophthalmic eponyms in the title of PubMed indexed documents per year since 1900 . Middle - the proportion of PubMed indexed documents using one of 291 ophthalmic eponyms in their title since 1900. Bottom - the ratio of PubMed indexed documents using one of 291 ophthalmic eponyms in their title to those using one of 50 common clinical terms since 1900
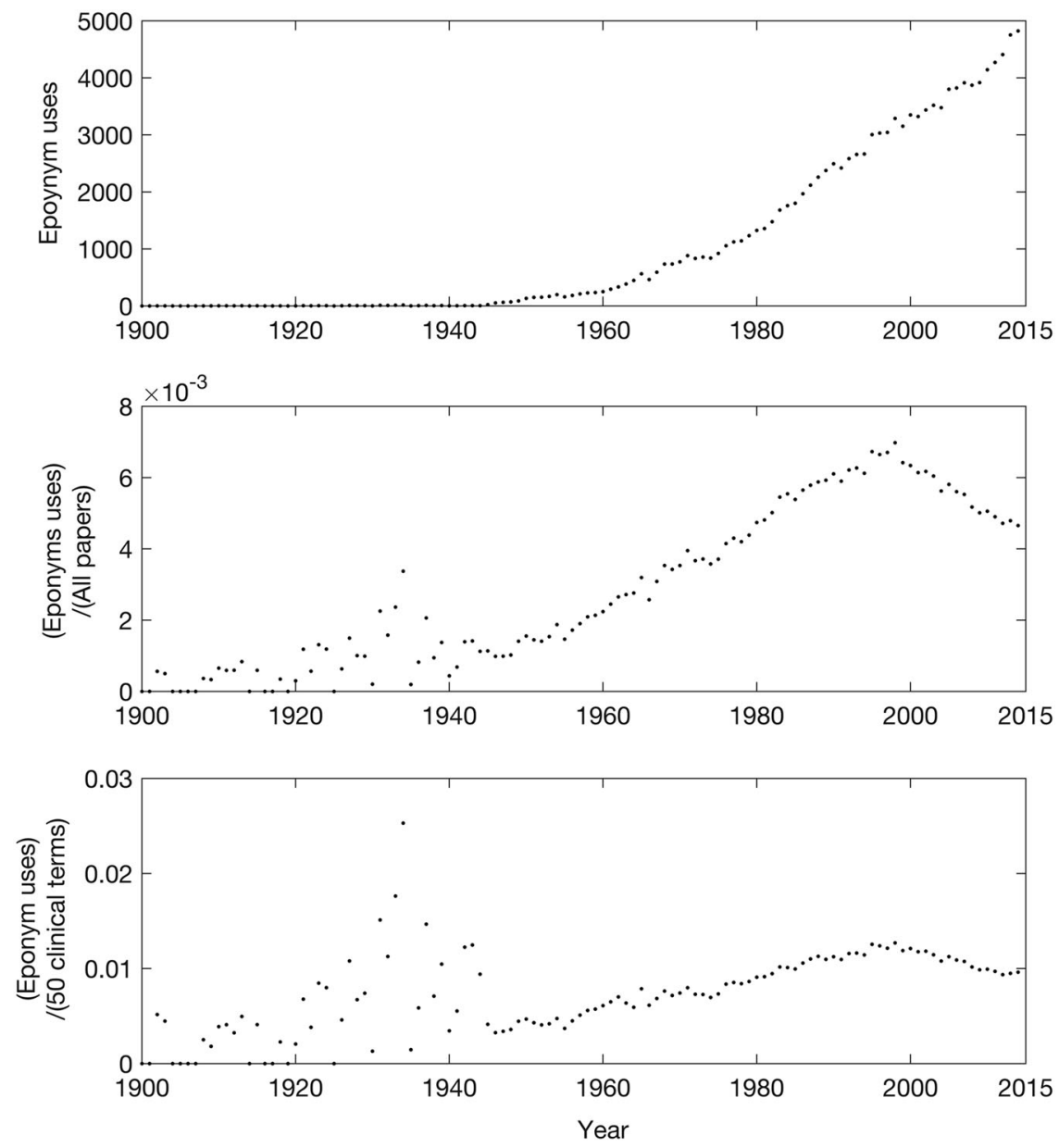

\title{
Response to: Comment on: Eponymous women in ophthalmology: syndromes with prominent eye manifestations named after female physicians
}

\author{
Sarah H. Van Tassel ${ }^{1} \cdot$ Kira L. Segal $^{1} \cdot$ Jessica B. Ciralsky ${ }^{1} \cdot$ Grace Sun $^{1}$
}

Received: 27 September 2018 / Accepted: 27 September 2018 / Published online: 18 December 2018

(c) The Royal College of Ophthalmologists 2018

We thank Thomas and Gunasekera for their interest in our article [1], the premise of which is to celebrate the achievements of pioneering women in ophthalmology.

Sarah H. Van Tassel

sjh2006@med.cornell.edu

1 Department of Ophthalmology, Weill Cornell Medicine, 1305 York Avenue, 11th floor, New York, NY 10021, USA
Empirical evidence demonstrates that eponym extinction overtook new eponym coinage in the 1980s, and the trend continues [2]. Eponym prevalence in journal article titles has been in decline since the early 1990s [2]; the letter from Thomas and Gunasekera affirms this observation with ophthalmic eponyms as well. These trends are in part due to the elimination of eponyms associated with Nazi physicians. Additionally, the eponymous David Cogan himself 
and others have called for reduced eponym coinage and use in favor of descriptive terms [3].

Eponyms commonly recognize one or two people, which may reflect influence, chance, seniority, politics, gender, or language rather than bearing witness to global discourse and collaborative scientific inquiry. For example, Tsuya Sakurai described the melanocytic iris hamartomas characteristic of neurofibromatosis type 1 [4], accompanied by her detailed illustrations, two years before Lisch's paper was published in the German language literature. Syndromes, the subject of our article, are particularly likely to be identified through the work of multiple individuals, each of whom describes clusters of signs and symptoms that may be subsequently identified as syndromic.

In the current era, an additional concern with eponyms is that varying use of the possessive and non-possessive forms is a challenge for search engines, which may produce incomplete disease-specific results depending on the term used [5]. This is a hindrance to scholarly research, medical writing, and information dissemination for patients and clinicians alike.

Eponyms honor contributors, serve as memory tools, and may be simpler than names routed in genetics, function, or symptoms (consider the eponymous lysosomal storage disorders). We agree with Thomas and Gunasekera that the decline in eponyms is not "precipitous," and there is no need to rapidly expunge eponyms from use. However, the empirical decline in eponym coinage as well as many authors' and investigators' reticence to eponyms suggests that future syndromic eponyms attributed to both male and female ophthalmologists will be rare. Thus, we recognize the historical contributions of the few women for whom ophthalmic syndromes are named.

\section{Compliance with ethical standards}

Conflict of interest The authors declare that they have no conflict of interest.

Publisher's note: Springer Nature remains neutral with regard to jurisdictional claims in published maps and institutional affiliations.

\section{References}

1. Van Tassel SH, Segal KL, Ciralsky JB, Sun G. Eponymous women in ophthalmology: syndromes with prominent eye manifestations named after female physicians. Eye Lond Engl. 2018;32:1293-5.

2. Thomas PB. Are medical eponyms really dying out? A study of their usage in the historical biomedical literature. J R Coll Physicians Edinb. 2016;46:295-9.

3. Cogan DG. The rise and fall of eponyms. Arch Ophthalmol. 1978;96:2202-3.

4. Sakurai T. Multiple neurofibroma patient showing multiple flecks on the anterior surface of the iris. Acta Soc Ophthalmol Jpn. 1935;39:87-93.

5. Ayesu K, Nguyen B, Harris S, Carlan S. The case for consistent use of medical eponyms by eliminating possessive forms. J Med Libr Assoc Jmla. 2018;106:127-9.

\title{
Comment on "Vitrectomy with scleral buckling versus with inferior retinectomy in treating primary rhegmatogenous retinal detachment with PVR and inferior breaks"
}

\author{
Upma Awasthi $^{1} \cdot$ Rohini Grover $^{1} \cdot$ Chetan Videkar $^{1} \cdot$ Abhishek Varshney $^{1}$ \\ Received: 21 November 2018 / Accepted: 29 November 2018 / Published online: 18 December 2018 \\ (c) The Royal College of Ophthalmologists 2018
}

Upma Awasthi

upma.awasthi2@gmail.com

1 Department of Vitreoretina, CL Gupta Eye Institute, Moradabad 244001 Uttar Pradesh, India
To the Editor

We read the article by Eleinen and Mohalhal [1] with great interest. We applaud them to do head on comparison of scleral bucklng (SB) and retinectomy (RR) as a primary approach in patients with rhegmatogenous retinal detachment (RRD) with proliferative vitreoretinopathy (PVR) with inferior breaks. However, we would like to comment upon few points. 